\title{
Profesor y portafolio en la consolidación de una cultura de la evaluación-como-aprendizaje ${ }^{1}$
}

\section{Teacher and Portfolio in the Consolidation of an Assessment-as-Learning Culture}

Edgar Picón-Jácome ${ }^{2}$

Citation/ Para citar este Artículo: Picón-Jácome, E. (2021). Profesor y portafolio en la consolidación de una cultura de la evaluación-comoaprendizaje. Colomb. Appl. Linguistic. J., 23(2), pp. 213-228.

Received: 09-Mar.-2020 / Accepted: 19-Aug.-2021

DOI: https://doi.org/10.14483/22487085.16004

\section{Resumen}

En este artículo se discuten los resultados de un estudio de caso en el que se exploraron los retos generados por la implementación de un portafolio evaluativo en el programa de inglés de una universidad pública colombiana. Los hallazgos surgidos del análisis de entrevistas semiestructuradas, grupos focales, reuniones de profesores y reflexiones de los participantes, seleccionados a partir de una muestra representativa, permitieron examinar la cotidianidad de sus prácticas evaluativas e identificar dinámicas, roles y conceptualizaciones relacionados con dicho proceso. Así mismo, develaron un impacto positivo para el desarrollo de la autonomía tanto en docentes como en estudiantes, derivado del ejercicio de una práctica reflexiva influida por el papel del profesorado en la implementación del portafolio. Sin embargo, se evidenciaron ciertas limitaciones en la viabilidad del proceso evaluativo a causa del tiempo y la experiencia que demanda una realimentación dialógica integrada a la enseñanza. Este reto fue resuelto por algunos profesores a través del uso de las TIC (Tecnologías de la Información y Comunicación) y al compartir sus experiencias, lo que muestra la importancia de articular la práctica docente y el desarrollo de habilidades tecnológicas a los programas de formación en evaluación.

Palabras clave: autonomía, evaluación, inglés como lengua extranjera, portafolio

\begin{abstract}
This article presents the results of a case study that explored the challenges of implementing an assessment portfolio in the EFL institutional program of a Colombian public university. The findings that resulted from the analysis of semi-structured interviews, focal groups, faculty meetings and teachers' reflections permitted entering the day-to-day evaluative practices of the participants, selected through a purposeful sampling approach, and identifying dynamics, roles and conceptualizations related to the portfolio implementation. The data analysis disclosed positive washback both on the development of teacher and learner autonomy, derived from the exercise of a teachers' reflective practice influenced by their role in the implementation of the portfolio. However, there was also evidence of practicality issues related to the time and experience demanded by a dialogic feedback integrated to the process vs. a focus on evaluating the product. Some teachers overcame this challenge through the use of

1 El presente artículo es resultado del proyecto: “Logros y retos del Sistema de Evaluación del PIFLE-l”, código 2018-20533, financiado por la Escuela de Idiomas de la Universidad de Antioquia a través de la "Convocatoria Formación en Lenguas Extranjeras", e inscrito en el Comité para el Desarrollo de la Investigación (CODI).

2 Universidad de Antioquia, Colombia. ORCID (D): https://orcid.org/0000-0002-6028-3925. edgar.picon@udea.edu.co.
\end{abstract}


ICT (Information and Communication Technologies) and the exchange of experiences. This revealed the importance of articulating teacher experience and ICT training to LAL (Language Assessment Literacies) programs.

Keywords: autonomy, assessment, English as a foreign language, portfolio

\section{Introducción}

\section{Antecedentes}

En el marco de la globalización y la reforma educativa y lingüística en Colombia (Usma Wilches, 2009), la Universidad de Antioquia instituyó en 2014 una nueva Política de Lengua Extranjera para sus estudiantes de pregrado. En esta política se crea el Programa Institucional de Formación en Lengua Extranjera-Inglés, PIFLE-I (Universidad de Antioquia, 2014). El artículo cinco del acuerdo determina: "Establecer como obligatorio, en los programas de pregrado que conduzcan a título profesional, la incorporación, en todos los planes de estudio, de cinco niveles de inglés", y su segundo parágrafo explicita como objetivo del programa el desarrollo de competencias comunicativas correspondientes a un nivel B1 del Marco Común Europeo de Referencia.

En consecuencia, se diseñó para el Programa un sistema que articula procedimientos evaluativos complementarios a través de un portafolio que actúa como eje transversal, fomentando el ejercicio de la coevaluación entre el estudiante y el profesor (Bratcher y Ryan, 2003; Picón-Jácome, 2012), y que contribuye a la consolidación de una cultura de la evaluación-como-aprendizaje ${ }^{3}$, disminuyendo así el potencial impacto negativo de la política en la vida académica de los estudiantes ${ }^{4}$.

3 Assessment as learning es un concepto acuñado en la literatura de evaluación en lengua inglesa. En este artículo, a la traducción al español del término se le añadieron los guiones para enfatizar la interacción de los términos evaluación y aprendizaje, además de definirlo como cultura (ver los referentes conceptuales).

4 Puesto que el inglés estaba siendo incluido en todos los pregrados de la universidad con sus respectivos créditos, perder uno de los cursos en tres ocasiones provocaría que un estudiante quedara por fuera de la institución, según lo estipulado en la norma.

\section{Planteamiento del problema}

El PIFLE-I significa un cambio positivo para los pregrados de la universidad, pero también presenta grandes retos:

1. Los cinco cursos que componen el Programa están integrados al currículo con créditos específicos, lo que constituye un componente de peso considerable dentro del pensum.

2. Una evaluación de carácter formativo y potenciadora de la autonomía como la que el PIFLE-I promueve representa un cambio de paradigma que demanda tiempo y apoyo para los profesores por parte de la administración.

3. Siendo el portafolio el espacio en el que la función formativa del sistema se hace operativa, evaluar su implementación es fundamental para comprender los retos que plantea la consolidación de una cultura de la evaluación como la que el PIFLE-I se ha propuesto llevar a cabo.

Identificar los retos que genera este cambio hace posible determinar acciones de apoyo específicas a las prácticas evaluativas de los profesores. Con ese fin se planteó un estudio de caso instrumental que determinó la utilidad (Bachman y Palmer, 1996) del sistema evaluativo y su impacto en dichas prácticas. En el contexto de dicho estudio se evaluó, a su vez, la implementación del portafolio, cuyos hallazgos se discuten en este artículo a partir de la pregunta: ćqué logros y retos en la consolidación de una cultura de la evaluación-como-aprendizaje se evidencian en la implementación del portafolio PIFLE-I?

\section{Referentes conceptuales}

En esta sección se definen los conceptos fundamentales que sustentan el estudio: la cultura de la evaluación-como-aprendizaje, el portafolio evaluativo, el impacto en la evaluación y la viabilidad.

\section{La cultura de la evaluación-como- aprendizaje}

En general, el término cultura se define como un conjunto de costumbres, ideas, 
conocimientos, normas, creencias, hábitos y capacidades que subyacen en el comportamiento social de un grupo (Merriam-Webster, n.d.). De manera más específica, Kramsch asevera que a menudo la cultura se percibe como "las actitudes y creencias comunes que una sociedad comparte, y los productos que dicha comunidad ha creado" (citado por Inbar-Lourie, 2008, p. 285). ${ }^{5}$ En la literatura referente al campo de la evaluación en lenguas los autores distinguen entre dos tipos de culturas: la cultura del testing -que podríamos traducir como "evaluación tradicional o de pruebas de papel y lápiz", con una función fundamentalmente sumativa- y la cultura del assessment -o de la "evaluación continua con una función fundamentalmente formativa", basada en el constructivismo (Inbar-Lourie, 2008). Enmarcada dentro de la cultura del assessment, en el PIFLE-I se pretende consolidar una cultura de la evaluación-como-aprendizaje, que en este artículo se define desde cuatro conceptos teóricos: la evaluación complementaria, la evaluación para el aprendizaje, la evaluación para la autonomía y la evaluación dinámica.

Shohamy (1998) enfatiza en el hecho de que un constructo complejo como la habilidad lingüística requiere para su evaluación de la aplicación de diferentes procedimientos complementarios, con base en lo que la autora denomina una perspectiva múltiple. En ella, se define la utilización de un procedimiento u otro dentro de un sistema evaluativo por su pertinencia en función de unos propósitos establecidos y la coherencia que debe existir entre el qué -el constructo a evaluar-y el cómo -el procedimiento evaluativo-. Por su parte, Wiliam (2011), siguiendo a los autores que cuestionan la dicotomía evaluación sumatival evaluación formativa, señala que una evaluación de carácter sumativo puede servir para funciones formativas, mientras que una evaluación formativa puede servir también para funciones sumativas. Según él, lo que define una evaluación para el aprendizaje es en realidad el propósito que la guía y, por consiguiente, cómo se utilizan los resultados para potenciar el aprendizaje; en otras palabras, el tipo de realimentación o feedback. Estos dos

5 Todas las traducciones que se presentan en este artículo son del autor. conceptos determinan la validez del sistema según Shohamy- y su impacto -de acuerdo con William-.

Por su lado, Lamb (2010) encuentra que una evaluación pensada para el aprendizaje está diseñada, a su vez, "para desarrollar [en los estudiantes] las capacidades necesarias para volverse aprendices autónomos bajo la perspectiva de mejorar el aprendizaje, a través de un mejor auto-monitoreo y auto-evaluación que los conduzca a una mejor planeación" (p. 101). Otros autores (Earl, 2006, 2013; Earl et al., 2015) Ilaman a este enfoque evaluación como aprendizaje y enfatizan en el desarrollo de la autorregulación de los estudiantes como uno de sus resultados más importantes. El auto monitoreo, la autoevaluación y la planeación son estrategias metacognitivas (Oxford, 2001) consistentes con una dimensión técnica de la autonomía del estudiante, aunque esta última también comprende una dimensión psicológica y una política (Benson, 1997). La dimensión psicológica, relacionada con el desarrollo de valores tales como la responsabilidad, la honestidad y el pensamiento crítico, se potencia mediante la coevaluación, que siguiendo el principio de zona de desarrollo potencial (ZPD) de Vigotsky posibilita la mediación; se habla entonces de evaluación dinámica (Poehner et al., 2017). La dimensión política, relacionada con la habilidad de los estudiantes para participar en la toma de decisiones que los afectan, se desarrolla en la medida que los estudiantes intervienen el proceso evaluativo. Aquí el docente juega un papel vital como guía autónomo (Little, 1995) al involucrar a los estudiantes activamente en el proceso, lo que fomenta procedimientos evaluativos más democráticos y justos (Gipps, 1999; PicónJácome, 2013; Shohamy, 2001; Suskie, 2002).

Así, siguiendo a los autores mencionados, la evaluación-como-aprendizaje puede definirse como un enfoque de evaluación dinámico integrado a la enseñanza que se concreta en un sistema articulador de procedimientos evaluativos complementarios pensados para potenciar el aprendizaje a través de la realimentación constructiva entre los actores que intervienen en el proceso, y que se alimenta del ejercicio autónomo de 
todos los involucrados. Pero para generar este tipo de evaluación es necesario entender la autonomía del estudiante como un principio integral, es decir, partiendo de una perspectiva que comprenda las dimensiones técnica, psicológica y política a las que Benson (1997) hace referencia.

\section{El portafolio evaluativo}

La inclusión del portafolio en el PIFLE-I responde a la importancia que se le da en el Programa al carácter formativo de la evaluación. El portafolio ha sido señalado como el instrumento evaluativo formativo por excelencia (Fox, 2008; Genesee y Upshur, 1996) debido a la posibilidad que ofrece para fomentar la reflexión y el ejercicio autónomo de los estudiantes, y establecer un diálogo con el docente.

De acuerdo con O'Malley y Valdez (1996), el portafolio es un procedimiento evaluativo auténtico y válido donde el desempeño del estudiante se evalúa conforme a las metas y actividades de clase, y sus contenidos representan lo que los estudiantes hacen en el aula, reflejando su progreso en relación con los objetivos de aprendizaje (p. 35). Los portafolios pueden ser de exhibición, de colección y evaluativos. En este último se deben incluir "las reflexiones enfocadas en objetivos específicos de aprendizaje que contienen una colección sistemática del trabajo de los estudiantes, la autoevaluación de los estudiantes y la evaluación del profesor" (p. 37). El portafolio PIFLE-I contiene esos tres elementos esenciales además de un espacio para la coevaluación negociada entre los estudiantes y el profesor.

El papel del portafolio en el PIFLE-I se enfoca, por lo tanto, en dos objetivos principales: (1) consolidar la articulación e integración de los procedimientos que componen su sistema evaluativo desde de una perspectiva múltiple y sistémica de la validez, y (2) abrir espacios de diálogo y reflexión por parte de los actores a lo largo del proceso, permitiendo el desarrollo y ejercicio de su autonomía. En resumen, el portafolio en el PIFLE-I es el eje transversal articulador del sistema evaluativo y se espera que actúe, no tanto como procedimiento, sino como un espacio de encuentro entre el profesor y sus estudiantes para la consolidación de una cultura de la evaluacióncomo-aprendizaje.

\section{El impacto}

El impacto en la evaluación se refiere al efecto que esta tiene en los individuos (a nivel micro) y en el currículo o la sociedad en general (a nivel macro). Cuando el impacto se da en los procesos de enseñanza y aprendizaje se habla de efecto washback (efecto de arrastre), que puede afectar el aprendizaje de los individuos, las prácticas de enseñanza de los profesores y los sistemas evaluativos en general, y puede ser tanto positivo como negativo (Bachman y Palmer, 1996). El washback positivo se refiere a un impacto deseable en estos procesos y su vehículo es la realimentación o feedback. Por ejemplo, se esperaría que una evaluación de naturaleza formativa tuviera un impacto positivo en el aprendizaje de los estudiantes y en las prácticas de enseñanza de los profesores en la medida en que unos y otros aprendieran del evento evaluativo. Autores como Brown y Hudson (1998) afirman que un sistema evaluativo articulado al currículo tiene como efecto un impacto o washback positivo, por lo que recomiendan alinear los procedimientos evaluativos a los objetivos y contenidos del programa. Dicha alineación, en consonancia con los fundamentos teóricos en los que se basa un determinado programa y en coherencia con las prácticas de los profesores, es lo que otros autores han denominado validez sistémica (Arias et al., 2009, 2012; Maturana, 2015).

\section{La viabilidad}

Según Bachman y Palmer (1996) la viabilidad (practicality) de la evaluación se refiere a la posibilidad de que esta se dé teniendo en cuenta los recursos disponibles en términos de tiempo, dinero, equipos, espacios físicos y mano de obra. De acuerdo con Brown (2004), una prueba es viable si "no es excesivamente costosa, se mantiene dentro de limitaciones de tiempo apropiadas, es relativamente fácil de aplicar y tiene un procedimiento de valoración y calificación que es específico y eficiente en cuanto al tiempo" (p. 19). 
La literatura de evaluación, en general, define la viabilidad en el contexto de las pruebas tradicionales estandarizadas (testing), por lo que -en el marco este estudio- es necesario comprenderla dentro del ámbito del aula. Si bien en este contexto la evaluación no presenta mayores limitaciones en cuanto a recursos económicos o espacios físicos, sí puede plantear grandes retos en términos del tiempo y de la formación docente que requiere. En palabras de Brown:

Es necesario anotar que más tiempo y presupuestos institucionales considerablemente más altos se requieren para implementar y calificar evaluaciones que presuponen una valoración más subjetiva, más individualizada y una mayor interacción en el proceso de ofrecer feedback. La ganancia, sin embargo, viene con una realimentación más útil a los estudiantes, una potencial motivación intrínseca $y$, en últimas, una descripción más completa de las habilidades del estudiante. (2004, p. 14)

Como se infiere de la cita de Brown, el washback o impacto de la evaluación en el proceso de enseñanza-aprendizaje es el principio significativo más esperado de un sistema como el diseñado para el PIFLE-I. Es necesario, entonces, encontrar un balance entre el impacto y la viabilidad para que dicho sistema alcance los objetivos propuestos.

\section{Metodología}

Este proyecto de investigación se llevó a cabo a través de la metodología de estudio de caso instrumental (Stake, 1995), inscrita dentro de la tradición cualitativa en investigación (Creswell, 2003, 2007). Según Stake (1995), en un estudio de caso instrumental "el investigador se enfoca en un asunto o preocupación y entonces selecciona un caso vinculado para ilustrar el asunto" (p. 74). De esta manera, se consideró el sistema evaluativo del PIFLE-I como un caso instrumental en cuanto permitía entender mejor los aspectos que inciden en el diseño de sistemas válidos y confiables de evaluación en el aula de inglés como lengua extranjera, en general. Además, se exploraron los factores que potencian u obstaculizan el proceso de enseñanza-aprendizaje a partir de una evaluación de naturaleza complementaria y formativa como la que promueve el PIFLE-I en dicho contexto.

\section{Técnicas de recolección de datos}

En este artículo se discuten los hallazgos de los datos arrojados por técnicas cualitativas en las que se exploraron las percepciones de los profesores; estas incluyeron: entrevistas semiestructuradas a dos profesores del Comité de Servicios (ES) ${ }^{6}$, dos grupos focales (GF), dos reuniones de profesores citadas por la coordinación (RP) y algunas reflexiones hechas por los participantes de una estrategia de acompañamiento denominada Programa Mentor (PM).

\section{Participantes}

La selección de los participantes se hizo siguiendo la lógica de muestra representativa (Patton, 1990). Los participantes que aceptaron firmar el consentimiento informado -o lo dieron a través de la plataforma del Programa Mentor$\mathrm{y}$ de quienes se presentan evidencias en este artículo fueron en total veinticinco: dos profesores ocasionales del Comité de Servicios, quienes participaron en el diseño e implementación del PIFLE-I (dictando cursos desde su inicio) y colaboraron en las entrevistas semiestructuradas; cuatro profesores vinculados y cinco ocasionales ${ }^{7}$ que también pertenecían al Comité, si bien no todos participaron en el diseño del Programa, pero sí en los grupos focales; y catorce profesores de cátedra ${ }^{8}$, en su mayoría recién ingresados al Programa, que tomaron el módulo de formación sobre el sistema evaluativo y se encontraban enseñando en cursos de diferentes niveles. Algunos profesores de cátedra participaron en grupos focales, otros dieron su

6 El PIFLE-I pertenece a la Sección de Servicios y Extensión de la Escuela de Idiomas, donde un comité conformado por los profesores de tiempo completo discute los asuntos curriculares relacionados con los programas de la Sección.

7 Los profesores de tiempo completo responden a dos figuras contractuales: los vinculados, con contratos a término indefinido, y los ocasionales, quienes son seleccionados para ocupar temporalmente algunas plazas de los vinculados.

8 Los profesores de cátedra prestan sus servicios a través de contratos por horas que se elaboran de acuerdo a la demanda de un programa específico. 
consentimiento en las reuniones de profesores y unos más compartieron sus reflexiones en el Programa Mentor.

Como miembro del Comité de Servicios, estuve a cargo de apoyar al PIFLE-I en sus procesos evaluativos $^{9}$ y durante la creación del programa diseñé su sistema de evaluación. Además, participé en el diseño del módulo de formación docente en evaluación y lo he impartido desde sus inicios. Finalmente, soy profesor del programa y en gran medida el presente artículo constituye un compromiso con mis colegas.

\section{Análisis, validación y consideraciones éticas}

Los datos obtenidos se analizaron teniendo en cuenta el marco conceptual para la creación de categorías deductivas y la generación de categorías inductivas surgidas de la lectura de las transcripciones correspondientes. Dichas categorías se validaron al interior del equipo investigativo siguiendo la técnica de cuatro etapas propuesta por Burns (1999), a saber: la organización de los datos, la categorización de la información, la comparación y reducción de las categorías, y la interpretación a partir de relaciones entre las categorías finales. Estos datos de naturaleza cualitativa se organizaron y categorizaron con la utilización del software NVivo (QSR International, 2002) y se interpretaron con la ayuda de tablas y árboles de categorías generados por el software.

Para garantizar un tratamiento ético de los datos recolectados se elaboraron consentimientos informados en los que se explicitó a los participantes las estrategias para garantizar su anonimato, los potenciales riesgos de su participación en el estudio y la libertad para retirarse en el momento que así lo consideraran. Cabe recordar que el estudio es un proyecto avalado por el CODI (Comité para el Desarrollo de la Investigación), organismo que regula la

9 El uso de la primera persona responde al compromiso ético de explicitar mi posicionamiento como parte de esta comunidad académica, mi papel como investigador participante y la responsabilidad que tengo de compartir los hallazgos con los docentes del programa. investigación en la Universidad de Antioquia, y aprobado en su momento por el Comité de Investigaciones y el Consejo de la Escuela de Idiomas de la misma universidad.

\section{Hallazgos y discusión}

Este estudio se enfocó en la comprensión de los logros y retos evidenciados en la implementación del portafolio PIFLE-I para la consolidación de una cultura de la evaluación-como-aprendizaje. Del análisis surgieron tres núcleos temáticos: el papel del profesor en la implementación del portafolio, el impacto del portafolio en la enseñanza y las conceptualizaciones del profesor sobre el portafolio. También es importante señalar que las voces de los participantes permitieron identificar el papel del profesor en la consolidación de dicha cultura y se presentan aquí como evidencia de sus prácticas, creencias, reflexiones, posiciones a favor $\mathrm{y}$ en contra, y emociones en general.

\section{Papel del profesor en la implementación del portafolio}

En este núcleo temático, que se divide en tres temas, a saber: guía y apoyo a los estudiantes, realimentación y valoración y calificación, se pueden identificar las actividades que los profesores llevan a cabo para la consolidación del proceso evaluativo que el portafolio requiere (ver Figuras 1, $\underline{2}$ y $\underline{3}$ ).

Desde el primer momento los profesores perciben desafíos inherentes al cambio de paradigma que el portafolio plantea. Su papel se lleva a cabo en dos dimensiones: una didáctica, relacionada con la organización del portafolio, y otra emocional, relacionada con la motivación de los estudiantes:

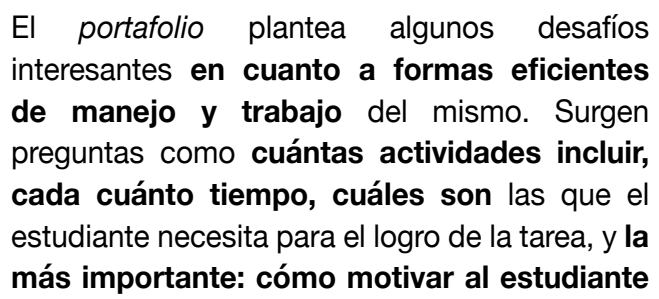




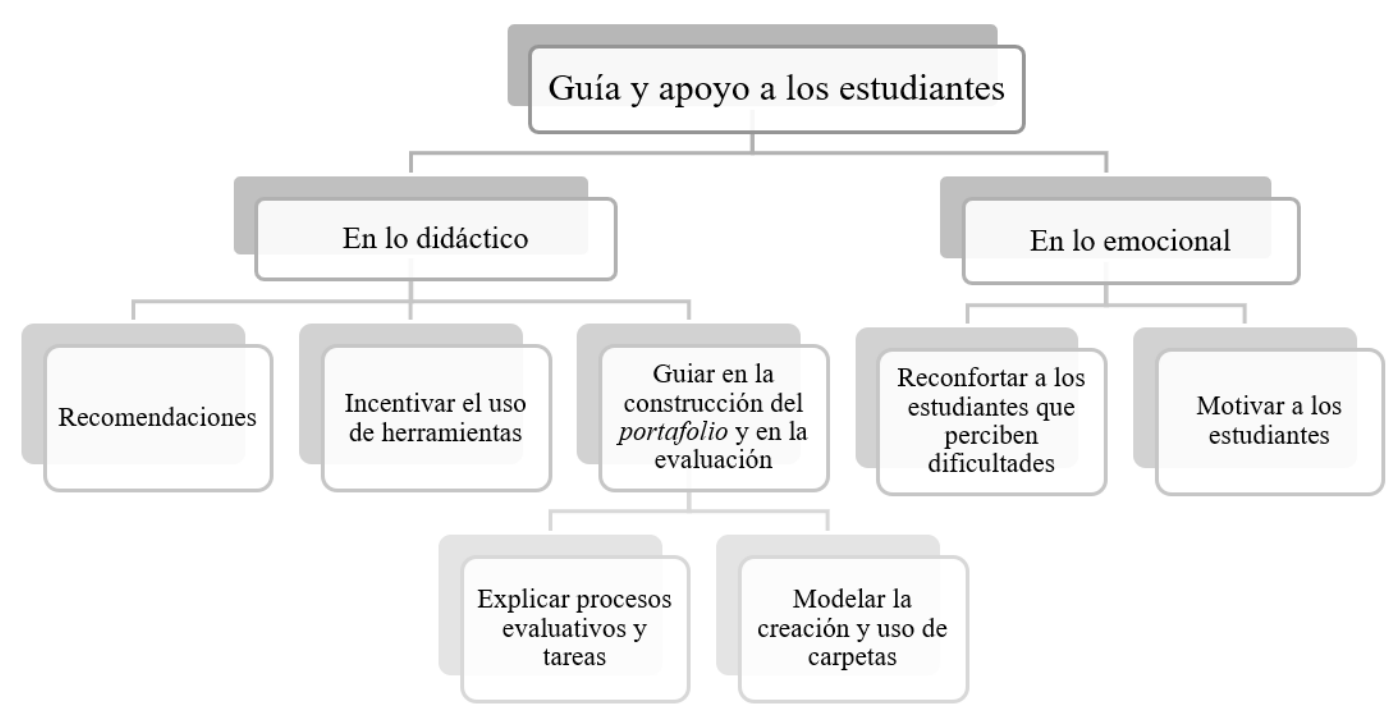

Figura 1. Papel del profesor: guía y apoyo a los estudiantes.

Fuente: elaboración propia.

en la selección de materiales que él mismo debe incluir en el portafolio y que den cuenta de su trabajo autónomo frente al logro de la tarea determinada. (PM, P8) ${ }^{10}$

El papel del docente como guía y apoyo se desarrolla con mayor intensidad durante las primeras unidades de cada curso y se vuelve fundamentalmente importante y demandante al inicio del programa:

Cuando yo llegué, uno se salió, otro se salió, ni siquiera me dijeron nada, solo me daba cuenta por el correo. Entonces me pongo a averiguar y reflexionar sobre mis procesos de enseñanza, les pregunté y un estudiante me dijo: "Profe, a mí me da tanto miedo lo que usted nos dijo, que yo preferí salirme", y eso pasa sobre todo con los del nivel 1. ¡A ellos les da tanto miedo todo! Por eso dicen cosas como: "con esta materia me puede bajar mucho el promedio y yo necesito subirlo, así

10 La citación de este y los próximos párrafos responde a la siguiente nomenclatura: Programa Mentor (PM), Grupo Focal (GF, 1 o 2 según el grupo), Reunión de Profesores (RP) y Comité de Servicios (ES). En seguida se incluye el número del participante, que para este caso sería Partipante 8 (P8) que prefiero salirme". Y pues eso es verdad, pero es que esos muchachos (los del primer nivel) vienen del colegio, escriben con rojo, escriben con negro, son todos lindos y ellos sienten demasiado miedo, sin embargo, yo lo que hago es incluir un poquito de español o de español-inglés. (GF2, P6)

Esta relación dialógica que se mueve entre lo racional y lo afectivo se vuelve crítica en los momentos de feedback. Los profesores implementan diferentes estrategias en una especie de ensayo y error, tratando de encontrar la forma más viable de dar realimentación (ver Figura 2):

[con] algunos [estudiantes] es corto y al grano, pero hay otros con los que hay que reflexionar más o el estudiante habla más y es difícil para uno como cortarlo. [A los estudiantes] con los que estoy muy de acuerdo con su autoevaluación, simplemente les pongo en el comentario "muy bien, sigue así, estoy de acuerdo." Llamo a algunos si de pronto hay algo que yo he visto y no estoy tan de acuerdo, aunque la verdad no pasa mucho. Podría asignar más tiempo para ellos y no para todos, porque algunos no lo necesitan (RP, P1). 


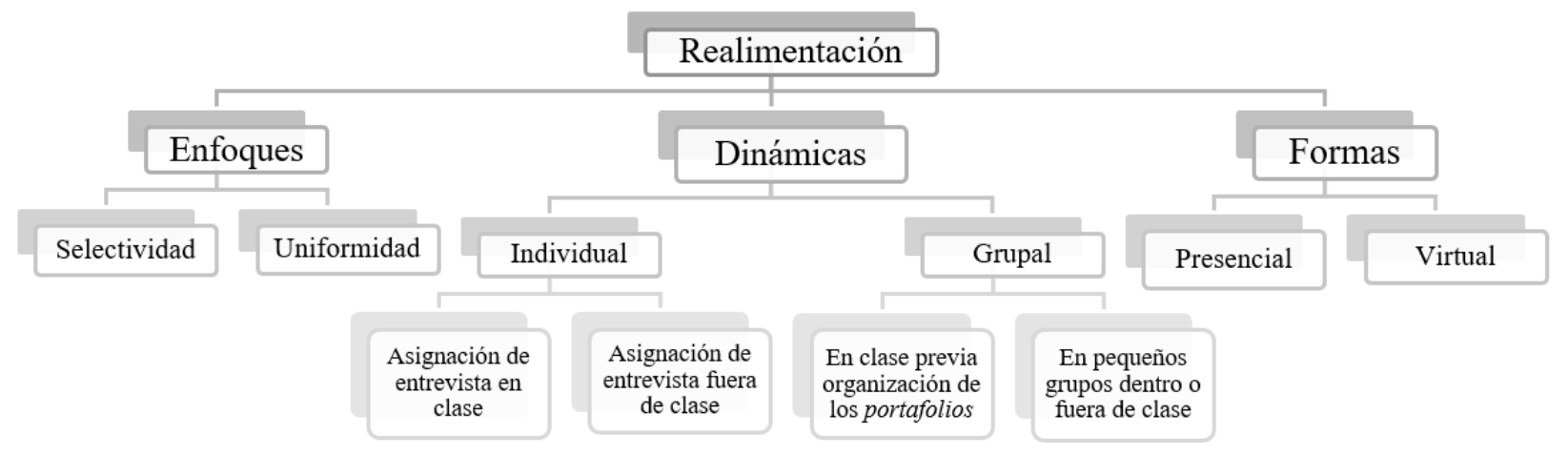

Figura 2. Papel del profesor: realimentación.

Fuente: elaboración propia.

La forma, la dinámica y el enfoque del feedback que el profesor selecciona se convierte en un momento potencial de impacto emocional debido al tiempo que demanda, afectando incluso la calidad de la realimentación:

Sentarlos de a uno en uno, no, sentía presión, que no me iba a dar el tiempo. Llegaba un momento en que el estudiante estaba hablando y yo lo veía moviendo la boca, entonces pensaba que se estaba acabando el tiempo, se iba a acabar la clase, faltaban los otros y yo era como "ajá, ajá, ok, listo", por lo que sé que esa estrategia no. (RP, P3)

El tiempo se señala específicamente como un obstáculo para lograr una realimentación individualizada: "el feedback personalizado para cada entrega de portafolio es una actividad que requiere una altísima dedicación personal y de horas, tanto para los estudiantes como para el docente, sea dentro o fuera de la clase" (PM, P4). Sin embargo, algunos profesores logran manejar el tiempo con éxito integrando la tecnología:

Entonces los estudiantes consignan ahí y los profesores entran y revisan. Ellos hicieron un google form para la autoevaluación y la rúbrica, y por ahí mismo les mandan la calificación a los estudiantes, quienes van a su autoevaluación. Esto me pareció muy práctico, creo que en términos de tiempo le puede ayudar mucho al profesor para organizarse. (RP, P5)
La viabilidad se incrementa al integrar el feedback al proceso de enseñanza:

Lo que hemos hecho con el portafolio en físico es que, cuando llegamos al salón, todos traen su portafolio, y como ya hemos hecho trabajos, o sea, cada evidencia que hay en el portafolio ya se ha trabajado en clase o ya se ha revisado, lo que hacemos en el aula es mirar cómo lo organizó y qué reflexión realizó el estudiante, y compartimos la reflexión. Ahí mismo se hace la rúbrica, se recoge y voilá, ya lo hicimos en una clase, ya calificamos. (RP, P4)

El análisis permitió identificar cambios en la relación de poder profesor-estudiante. Cuando esto sucede, el momento para el feedback se torna un espacio reflexivo, más constructivo, que genera empoderamiento:

Pero en la manera en que voy dándoles la responsabilidad a ellos [los estudiantes] y no tomándola yo, siento que el proceso se vuelve más amigable y sigue siendo muy formativo. (RP, P1)

Yo le digo al estudiante: "organiza el portafolio como si fuera tu hoja de vida y tuvieras que mostrarla aquí [...]lo que vamos a hacer es que te vayas haciendo cargo de lo que necesitas para aprender inglés, y que te sientas y reflexiones sobre qué es lo que lograste y qué es lo que te propones lograr en el siguiente paso del portafolio y al final del semestre, etc". (RP, P4) 


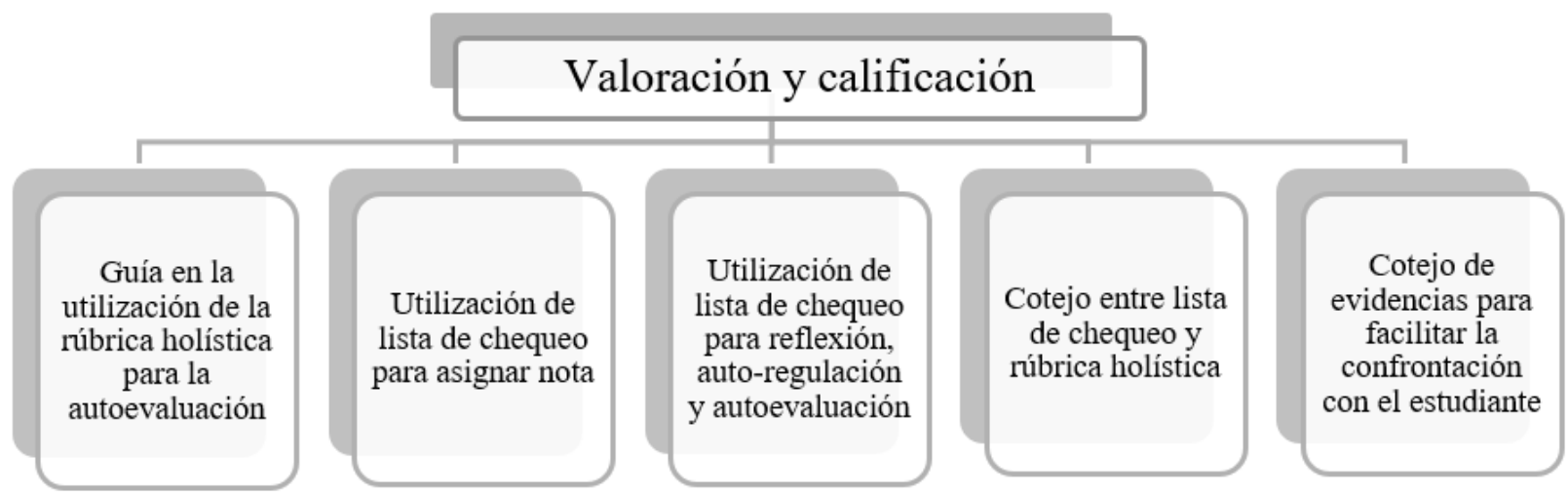

Figura 3. Papel del profesor: valoración y calificación.

Fuente: elaboración propia.

El proceso de realimentación lleva indistintamente al momento de valoración y calificación. En la práctica, los profesores afrontan este evento de maneras diferentes (ver Figura 3).

Aunque el portafolio está pensado para fomentar el compromiso de los estudiantes con su proceso de aprendizaje, algunos profesores se enfocan exclusivamente en lo lingüístico al momento de calificar, y parecen mantener el control. Otros guían la reflexión de los estudiantes para fomentar la autorregulación:

\begin{abstract}
Algunos le ponemos bastante cuidado al checklist ${ }^{11}$, pues informa dónde se ubica el estudiante en términos lingüísticos. $Y$ esto lo tenemos muy en cuenta en el momento de dar la calificación del portafolio. (RP, P5)

En la [check-list] yo no utilizo esta parte de los criterios lingüísticos para hacer la evaluación [calificación] que se va a dar del portafolio, sino para ver el plan que los estudiantes van a hacer, porque aquí es donde se identifican las dificultades que han tenido. [...] Al final ellos ponen las fortalezas y los aspectos a mejorar [...]. Y con esa reflexión hay que hacer el plan de trabajo de acuerdo a cómo ellos se evaluaron en lo lingüístico. (RP, P3)
\end{abstract}

Llama la atención que los participantes no se refirieron a la presentación de las escalas evaluativas

11 La rúbrica del portafolio contiene una escala cualitativa conocida como check-list para que los estudiantes se autoevalúen frente al logro de los objetivos lingüísticos de la unidad respectiva. como parte de su rol, lo que deja la duda de qué tanto las comprenden y utilizan durante el proceso, y no solo al final. En resumen, este núcleo temático permitió observar algunos de los asuntos que un cambio de paradigma suscita en el quehacer de los profesores; asuntos que en buena medida se enmarcan en lo que podríamos denominar el impacto y que constituye el tema central del siguiente núcleo.

\section{Impacto del portafolio en la enseñanza}

Teniendo en cuenta que este artículo también analiza el impacto del contexto en el aula de clase, estamos hablando del efecto de arrastre o washback (Bachman y Palmer, 1996). Por tanto, aquí se discute dicho efecto en las prácticas de enseñanza de los profesores como resultado de la implementación del portafolio. Las percepciones de estos sobre el impacto del portafolio para el aprendizaje se tratan como factor que influye en su inversión ${ }^{12}$ respecto a la propuesta evaluativa del PIFLE-I (ver Figuras 4 y $\underline{5}$ ).

El análisis permitió identificar un impacto negativo del portafolio en la enseñanza relacionado con el tiempo que requiere el tipo de realimentación que demanda. Para algunos, este asunto afecta de tal manera sus prácticas que desdibuja el valor de la evaluación como parte fundamental del proceso de enseñanza-aprendizaje:

12 En este aparte se utiliza el término inversión, desarrollado por Norton (1995), para describir la relación de interacción entre el profesor y la propuesta evaluativa. 
La verdad, sí es abrumadora la cantidad de evaluaciones que se tienen en el Programa, y a veces uno piensa si no es mejor hacer lo de muchos profesores que, o bien dejan por fuera algunas cosas y las reemplazan con otro tipo de actividades no evaluativas, o bien hacen las cosas muy rápido y por ende no son significativas para los estudiantes ni para el profesor. Es complejo todo ese tipo de evaluaciones y uno está siempre sobre el tiempo para aplicar las actividades. (GF1, P12)

Para estos profesores el portafolio se percibe como una pérdida de tiempo que los lleva a desistir de su implementación:

En mis cursos les he preguntado a los estudiantes sobre el portafolio. Entre otras respuestas, la más recurrente es que piensan que el portafolio es "una perdedera de tiempo", en palabras de ellos. Además, hay comentarios sobre si es útil o no hacerlo (sí, si aprenden algo cuando lo hacen), y sobre todo la relación que este tiene con otras actividades. (GF1 P 3)

De lo anterior se infiere que son los estudiantes los que determinan el valor del portafolio y los que deciden hacerlo o no, cuando en realidad es responsabilidad del profesor llevarlo a cabo.
A pesar de esta limitación -e incluso gracias a ella- uno de los logros más importantes fue el fomento de una práctica evaluativa reflexiva:

Para mí el portafolio ha significado repensarme como docente desde diferentes perspectivas porque me ha mostrado una posibilidad muy valiosa para hacer crecer a mis estudiantes a partir de sus intereses, sus inquietudes y sus propios procesos de aprendizaje. (PM, P1)

La mayoría reconoce el impacto positivo del portafolio en el aprendizaje, lo que es fundamental para lograr que inviertan en el cambio:

\begin{abstract}
Los beneficios han sido muchos. Mediante esta forma de evaluación los estudiantes pueden reconocer más fácilmente sus debilidades y fortalezas en un tema determinado, y ello es fundamental para su motivación. A su vez, les permite evidenciar sus logros y progresos en diferentes aspectos, como el vocabulario, la gramática y las estructuras, lo que les resulta sorprendente, pues ellos saben que tienen debilidades pero muchas veces no reconocen cuáles son. (PM, P3)
\end{abstract}

Una evaluación-como-aprendizaje implica un canje de roles en el que los estudiantes van adquiriendo mayor responsabilidad con el apoyo del profesor:

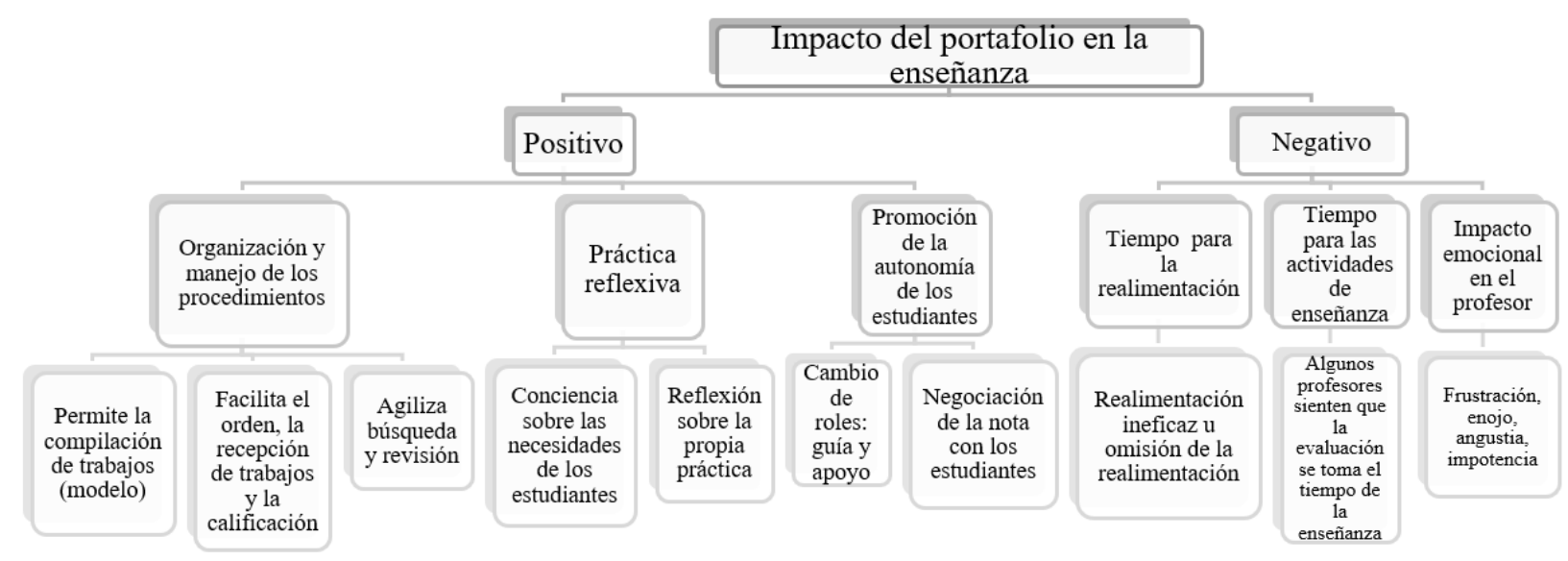

Figura 4. Impacto del portafolio en la enseñanza.

Fuente: elaboración propia. 


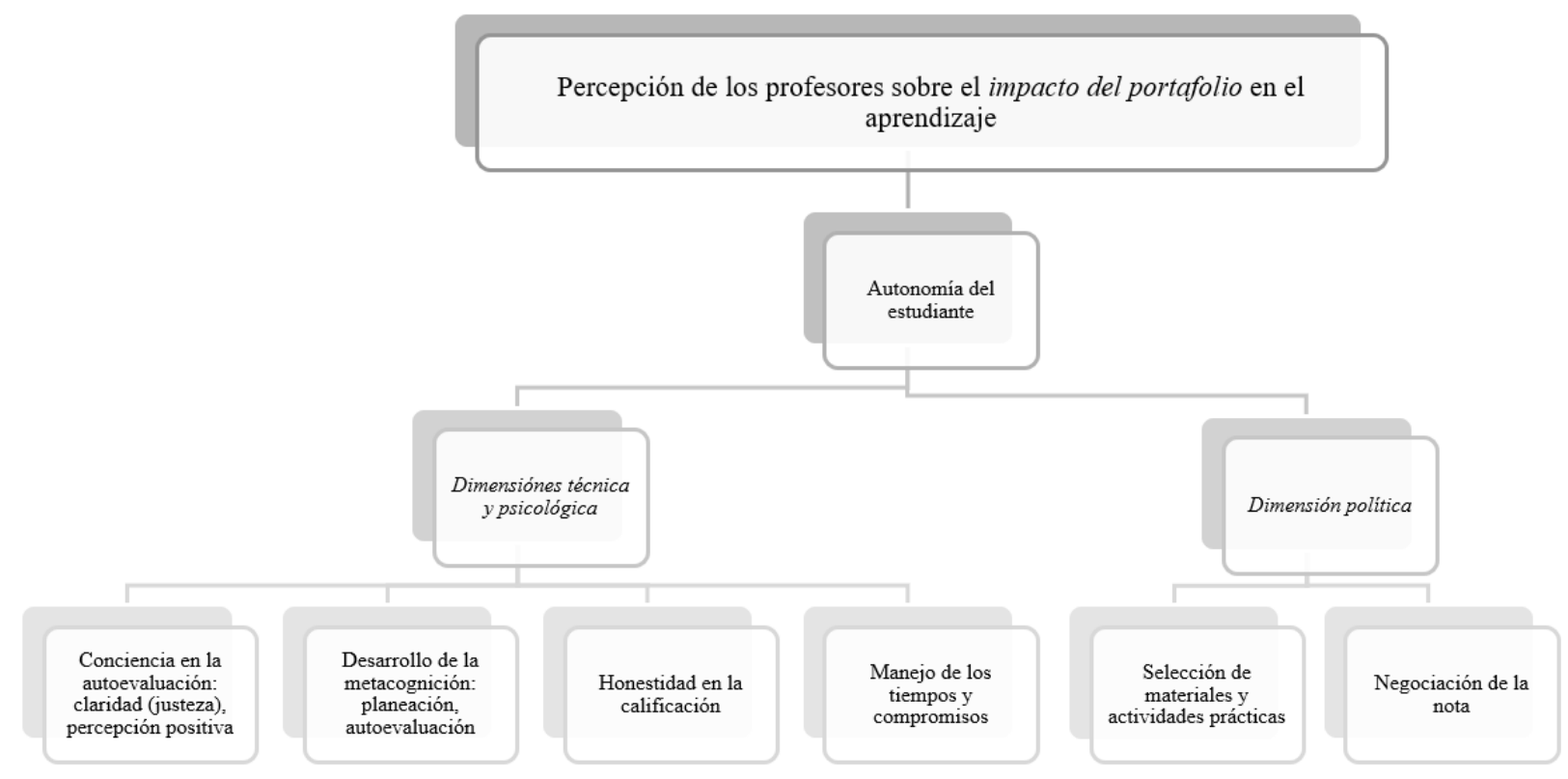

Figura 5. Percepción de los profesores sobre el impacto del portafolio en el aprendizaje.

Fuente: elaboración propia.

Para los estudiantes, desde mi experiencia y considerando los comentarios que hacían, es importante comprender que ellos son los responsables de su propio proceso de aprendizaje, que el docente es un orientador, pero que son ellos quienes en realidad "terminan poniendo la nota que merecen" (así lo decían) en la medida en que desarrollan un proceso de trabajo autónomo, a su ritmo, acorde con sus capacidades y necesidades. (PM, P8)

Por último, los profesores logran ver los beneficios y dan cuenta del desarrollo y ejercicio autónomo de sus estudiantes: "Al final, vi que muchos lograron un nivel más alto de conciencia, compromiso y responsabilidad. Las notas que se colocaron en el portafolio tuvieron comentarios o soportes muy claros y precisos frente a la rúbrica" (PM, P3).

En resumen, para lograr la consolidación de una propuesta de evaluación formativa como cultura se requiere que los profesores comprendan y valoren la importancia de su papel:
El portafolio es algo muy grande [...] yo creo que es una herramienta muy rica y hay que trabajarle mucho, hay que ponernos mucho más serios frente a todo lo que realmente implica y las bondades que tiene [...] porque si yo no le veo muchas, el estudiante le va a ver muchas menos. (GF1, P7)

El análisis develó, así mismo, diferentes comprensiones en los profesores sobre lo que es el portafolio; comprensiones que se presentan a continuación como sus conceptualizaciones.

\section{Conceptualizaciones del profesor sobre el portafolio}

El término conceptualización se refiere a las ideas que los profesores gestan a partir de su experiencia en la implementación del portafolio PIFLE-I y de la comprensión que logran del mismo. Podría decirse que son representaciones abstractas de lo que este significa para ellos dentro de sus prácticas evaluativas (Diccionario actual, n.d.). El análisis evidencia, entonces, diferencias en la conceptualización que los participantes tienen del portafolio, integradas en dos 
patrones (ver Figura 6). Estas diferencias parecen estar relacionadas con su formación y experiencia dentro del Programa, por lo que es necesario considerar estos aspectos en la discusión:

En el primer patrón los profesores perciben el sistema de evaluación como una especie de camisa de fuerza. Esto lo relacionan con el hecho de que hay actividades de clase preestablecidas y sienten que no pueden integrar su experiencia docente al proceso de construcción de la tarea comunicativa:

-P5: ¿Y cómo sería si la clase no fuera con PIFLE? $\mathrm{O}$ sea, ¿cómo eran nuestras clases antes? ¿O al momento de la evaluación antes del PIFLE?

-P1: [Había] más libertad de cátedra porque tú estás preparando guías o actividades para que los chicos realmente aprendan [...] estoy de acuerdo con los contenidos y los temas [del PIFLE], pero tendría [en otro programa] más libertad para planear y prepararme [...]. Entonces tú te encargarías de que los chicos aprendan a aprender, que no sientan que la guía ${ }^{13}$ hay que subirla al portafolio, sino que lo hacen porque

13 Algunos docentes se referían al material de apoyo como "las guías". Aunque realmente no estaba determinado así, muchos consideraban obligatorio que los estudiantes las incluyeran en el portafolio como evidencia de su trabajo. quieren, y no porque tienen que tener una nota en el portafolio. (GF2, P5 y P1)

En consecuencia, la tarea comunicativa ${ }^{14}$ se entiende como la única opción de evaluación y calificación válida, y aunque los profesores han guiado el proceso de construcción de tareas en otros contextos, no logran integrar su experiencia al portafolio:

Por ejemplo, en la preparación de la tarea yo sé que uno les pone nota, pero solo la evalúa al momento de la entrega. Uno como profesor puede pensar: "van a entregar un borrador, un ensayo o una plantilla", y uno lo puede cuadrar después. Pero sí hay una disparidad con la rúbrica en sí: no hay diversidad de opciones para evaluar el proceso de construcción de la tarea, no hay forma, simplemente es video entregado, video evaluado. Yo, en cambio, haría actividades de ensayo, preparación de talleres o evaluaciones conjuntas donde todos vean los videos de todos [...]. Pero como se está dando la rúbrica y la guía al momento de la entrega, no hay otras opciones. (GF2, P4)

14 Las tareas comunicativas son el corazón del sistema ya que el Programa se inserta en un enfoque TBLT.

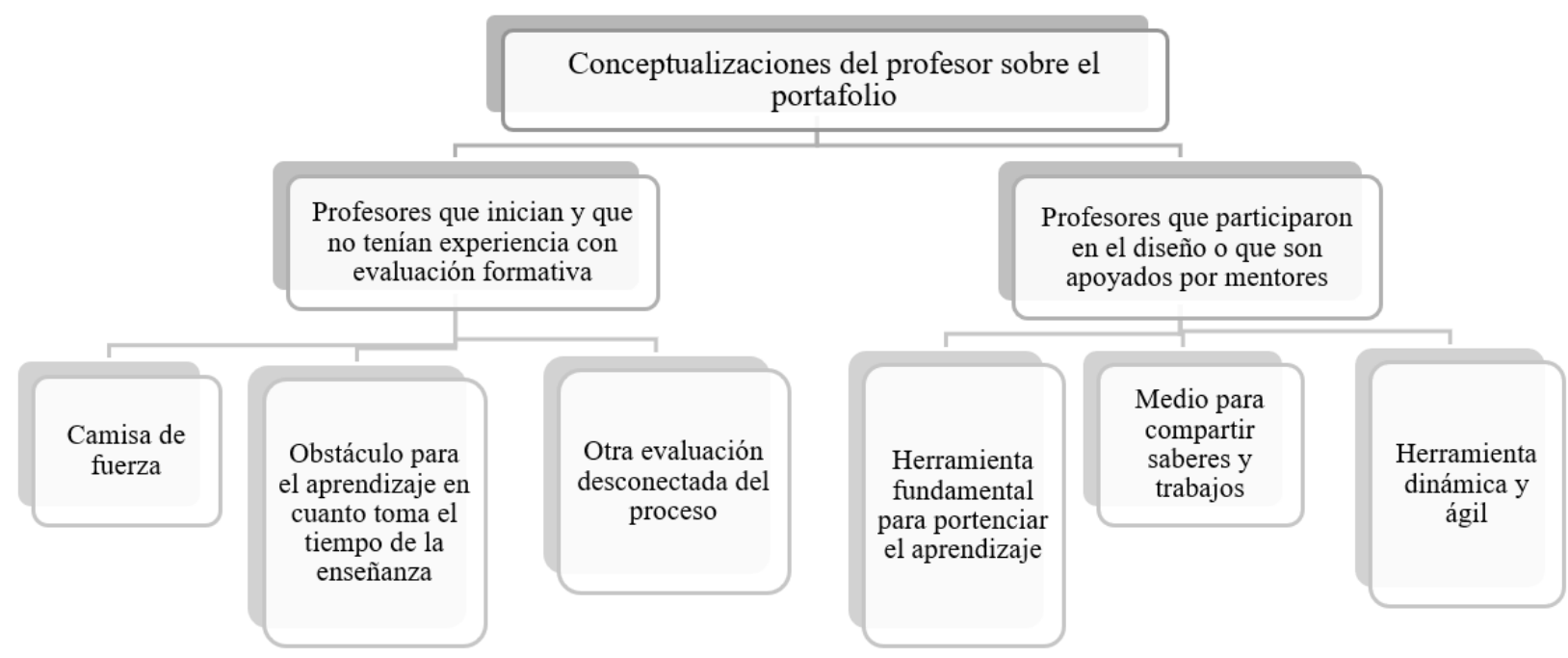

Figura 6. Conceptualizaciones del profesor sobre el portafolio.

Fuente: elaboración propia. 
Puesto que no ven la evaluación integrada a la enseñanza, los profesores se preguntan cómo acompañar el proceso.

En qué momento yo puedo acompañar al estudiante para que no llene la información el día anterior a revisar el portafolio, sino que sea como una consulta diaria o periódica [...] que se apropien, pero que no lo llenen al final, porque hay casos en que alguien no hizo nada y el día anterior llenó todo para sacar 5 , aunque yo no vi de dónde sacó esos 5. (GF1, P7)

Es como si el portafolio fuera una evaluación más del producto. El sistema se ve como un montón de actividades evaluativas que obstaculizan la enseñanza:

En lo personal, yo encuentro que es mucho contenido para un solo módulo y, como los otros profes dicen, son escasamente 16 semanas, de las cuales normalmente se pierde mucho tiempo. Adicional a eso se utilizan mucho para la evaluación, por lo que el aprendizaje del inglés y los temas en general no se pueden cubrir. (GF1, P5)

En el segundo patrón los profesores compartieron percepciones positivas sobre el portafolio, al que ven como una herramienta valiosa para lograr los objetivos esenciales que el sistema plantea:

El portafolio dentro de los cursos del Programa ha significado una herramienta de trabajo fundamental para el desarrollo y el logro de las tareas planteadas en el curso. De igual modo, representa un mecanismo de apoyo para el estudiante que le permite llevar a cabo los procesos de autorreflexión sobre las fortalezas y debilidades que asume en el desarrollo de las actividades dentro del curso. (PM, P3)

Con respecto al portafolio, creo que es una excelente herramienta y los estudiantes dan evidencias de su aprendizaje en los temas que se van tratando durante el curso. (GF1, P4)

Algunos manifiestan necesitar experiencia y apoyo para apropiarse de la herramienta; empero, no se expresan sobre el portafolio de manera negativa:

Lo que más me ha afectado es mi inexperiencia, sobre todo con estudiantes ique ni siquiera han visitado la universidad! Por eso, la formación también ayuda mucho; a nivel del profesor hay que considerar cómo lo afecta, cómo cambian sus prácticas y la manera de ver la evaluación. A veces me toca ir donde mi mentor y decirle: "yo necesito que usted me haga una hipnosis, porque yo mañana no sé qué hacer". ¡Yo no sabía qué había que hacer en la clase! Como me sentía totalmente perdida, él me contó mucho-y desde su experiencia- sobre los videos, cómo montarlos en el google classroom; me dijo [lo] qué tenía que decir en la primera clase, o sea, él me hizo el paso a paso [...] ahí entendí que esto también es de experticia, de seguridad, de formación. (GF2, P6)

Es claro, por tanto, que hay aspectos de la identidad del profesor que influyen en sus conceptualizaciones:

Siempre he considerado importante orientar estrategias de aprendizaje, y con el portafolio he encontrado un modo de hacerlo de manera más personalizada pues me ha permitido ser mucho más consciente de las necesidades académicas de mis estudiantes. Con el portafolio tuve la oportunidad de conocerlos mejor y de guiarlos de acuerdo con sus inquietudes, que muchas veces iban más allá de los contenidos del Programa. Por eso pienso que es una herramienta importante para hacer realimentación permanente y permitir que cada estudiante avance a su ritmo. (PM, P1)

La formación del profesor y su participación en el diseño del Programa parecen ser determinantes en el desarrollo de sus conceptualizaciones. Es evidente, por ejemplo, que las habilidades de algunos con la tecnología terminaron favoreciendo en ellos una actitud positiva hacia el portafolio, al que conceptualizan como una herramienta útil y ágil:

Lo que pasa es que yo tuve la fortuna de participar en el diseño de los cursos y eso me ayudó a 
estar enterado de lo que estaban haciendo los demás equipos, incluido el portafolio y el trabajo que se quería hacer con él. Y, además, porque tengo ciertas habilidades con las tecnologías que me permitieron adoptar rápido y fácil el uso del portafolio virtual [...]. A mí me gusta trabajar con el portafolio, me parece una herramienta dinámica y ágil. Me ha gustado, sobre todo que quede evidencia del trabajo hecho: porque hay unos trabajos muy buenos, que vale la pena mostrar a otros estudiantes de otros cursos como modelos de tarea [...]. Esto es algo que te permite ser más ordenado y, para mí, más ágil en la recepción de tareas y en la calificación también. (ES, P1)

A la postre, los profesores con mayor tiempo en el Programa han desarrollado una mayor comprensión sobre su papel y el papel del portafolio para el fomento de la autorregulación en los estudiantes. Esta comprensión, que ganan a partir de su experiencia y como testigos del proceso, genera en ellos claridad y compromiso en la implementación del sistema evaluativo:

Yo pienso que, aunque sea time consuming, sobre todo en los primeros niveles, es necesario hacer un acompañamiento a esa autoevaluación, que el docente realmente le saque el tiempo para sentarse con cada estudiante a explicarle, a preguntarle qué criterios tuvo él para hacer su autoevaluación, qué ingresó en ese portafolio, qué hizo en ese portafolio. Eso me parece primordial en los primeros niveles [...]. Cuando después estuve en el nivel 4 sentí que los estudiantes ya tenían muy interiorizado eso, tenían ya unos criterios mucho más claros frente a cómo autoevaluarse y de cierta manera eran muy transparentes en cómo lo hacían. Eran capaces de decir: "Profe, hice esto y me merezco esto, hice mucho más, -o- no hice nada, o hice demasiado poco, por consiguiente, me merezco esto". (ES, P2)

\section{Conclusiones}

Gracias al análisis se pudo explorar la cotidianidad del profesorado para comprender los retos que la implementación de un portafolio evaluativo representa. Se identificó que el profesor juega el papel de guía y apoyo para los estudiantes tanto en la organización y autoevaluación de sus portafolios como en la disminución del impacto emocional que tiene la evaluación para ellos. Los hallazgos permitieron describir el proceso de retroalimentación y categorizar diferentes enfoques, dinámicas y formas del mismo que el profesorado utiliza, y revelaron que el tiempo de la retroalimentación es el reto más importante que los profesores enfrentan.

Se identificaron, además, diferentes conceptualizaciones de los docentes sobre el portafolio y su utilidad, fundamentales en la comprensión de cómo y por qué la implementación de este procedimiento evaluativo les afecta. Algunos lo percibieron como una evaluación más y una camisa de fuerza, mientras que otros encontraron en él una herramienta valiosa para promover el aprendizaje, y una forma útil y ágil de organización del proceso. El análisis sugiere que dichas conceptualizaciones están influidas por la experticia de los docentes en el uso de las TIC, el apoyo entre los colegas y la formación profesional en evaluación, sustentando la efectividad de articular estrategias de formación docente que integren, a su vez, el compartir experiencias y desarrollar habilidades tecnológicas.

Finalmente, aunque al momento de la calificación quedó en relieve la dificultad que aún representa para algunos docentes superar un enfoque de evaluación numérica basada en el producto y enfocarse en la evaluación como proceso, el análisis mostró que el portafolio influye positivamente en el desarrollo de la autonomía de los profesores en la medida en que les demanda formación, genera el desarrollo de habilidades, promueve su reflexión sobre la práctica y los motiva a abrir espacios para el empoderamiento de los estudiantes. Este hallazgo ratifica el carácter interdependiente -de la relación estudiante-profesor- que hay en la autonomía y evidencia un giro en la práctica evaluativa dentro del Programa hacia una cultura de la evaluacióncomo-aprendizaje, que como todo cambio genera resistencia y requiere tiempo. 


\section{Referencias}

Arias, C., Estrada, L., Areiza, H. y Restrepo, E. A. (2009). Sistema de evaluación en lenguas extranjeras. Universidad de Antioquia.

Arias, C., Maturana, L. y Restrepo, M. I. (2012). Evaluación de los aprendizajes en lenguas extranjeras: hacia prácticas justas y democráticas. Lenguaje, 40(1), 99-126.

Bachman, L. F. y Palmer, A. S. (1996). Language testing in practice: designing and developing useful language tests. Oxford University Press.

Benson, P. (1997). The philosophy and politics of learner autonomy. En P. Benson y P. Voler (Eds.), Autonomy and independence in language learning (pp. 18-34). Addison Wesley Longman.

Bratcher, S. y Ryan, L. (2003). Evaluating children's writing: a handbook of grading choices for classroom teachers ( $2^{\mathrm{a}}$ ed). Routledge. https://doi. org/10.4324/9781410609069

Brown, H. D. (2004). Language assessment: principles and classroom practices. Longman.

Brown, J. D. y Hudson, T. (1998). The alternatives in language assessment. TESOL Quarterly, 32(4), 653675. https://doi.org/10.2307/3587999

Burns, A. (1999). Collaborative action research for english language teachers. Cambridge University Press.

Creswell, J. W. (2003). Research design: qualitative, quantitative and mixed methods approaches ( $\left.2^{\mathrm{a}} \mathrm{ed}\right)$. Sage.

Creswell, J. W. (2007). Qualitative inquiry and research design: choosing among five approaches ( $\left.2^{\mathrm{a}} \mathrm{ed}\right)$. Sage.

Diccionario actual. (n.d.). Conceptualización. En Diccionarioactual.com. Recuperado el 6 de octubre de 2021, de https://diccionarioactual.com/ conceptualizacion/

Earl, L. (2006). Assessment - A powerful lever for learning. Brock Education Journal, 16(1), 1-15. https://doi. org/10.26522/brocked.v16i1.29

Earl, L. (2013). Assessment as learning: using classroom assessment to maximize student learning. Corwin.

Earl, L., Volante, L. y DeLuca, C. (15 de junio de 2015). Assessment for learning across Canada. Education Canada Magazine. https://www.edcan.ca/articles/ assessment-for-learning-across-canada/

Fox, J. (2008). Alternative assessment. En E. Shohamy y N. H. Hornberger (Eds.), Encyclopedia of language and education (Vol. VII, Language testing and assessment) (pp. 97-109). Springer. https://doi. org/10.1007/978-0-387-30424-3_170
Genesee, F. y Upshur, J. (1996). Portfolios and conferences. En F. Genesee y J. Upshur (Eds.), Classroom-based evaluation in second language education (pp. 98117). Cambridge University Press.

Gipps, C. (1999). Chapter 10: socio-cultural aspects of assessment. Review of Research in Education, 24(1), 355-392. https://doi. org/10.3102/0091732x024001355

Inbar-Lourie, O. (2008). Language assessment culture. En E. Shohamy y N. H. Hornberger (Eds.), Encyclopedia of language and education (pp. 285-300). Springer. https://doi.org/10.1007/978-0-387-30424-3 182

Lamb, T. (2010). Assessment of autonomy or assessment for autonomy? Evaluating learner autonomy for formative purpose. En Paran y L. Sercu (Ed.), Testing the untestable in language education (pp. 98-119).

Little, D. (1995). Learning as dialogue: The dependence of learner autonomy on teacher autonomy. System, 23(2), 175-181. $\quad$ https://doi.org/10.1016/0346251X(95)00006-6

Maturana, L. (2015). Evaluación de aprendizajes en el contexto de otras lenguas. Fondo Editorial Funlam. https://www.funlam.edu.co/uploads/ fondoeditorial/89 Evaluacion_de_aprendizajes en el_contexto_de otras lenguas.pdf

Merriam-Webster. (n.d.). Culture. En Merriam-Webster. com dictionary. Recuperado el 6 de octubre de 2021, de https://www.merriam-webster.com/ dictionary/culture

Norton, B. (1995). Social Identity, Investment, and Language Learning. TESOL Quarterly, 29(1), 9-31. http://www.jstor.org/stable/3587803

O'Malley, J. M. y Valdez, P. L. (1996). Authentic assessment for English language learners: Practical approaches for teachers. Longman.

Oxford, R. (2001). Language learning styles and strategies. En M. Celce-Murcia (Ed.), Teaching English as a second or foreign language (3a., pp. 359-366). Heinle $\varepsilon$ Heinle.

Patton, M. Q. (1990). Qualitative evaluation and research methods (1a.). Sage.

Picón-Jácome, E. (2012). Promoting learner autonomy through teacher-student partnership assessment in an american high school: a cycle of action research. Profile: Issues in Teachers' Professional Development, 14(2), 145-162. https://revistas.unal. edu.co/index.php/profile/article/view/34070/34217

Picón-Jácome, E. (2013). La rúbrica y la justicia en la evaluación. Íkala, 18(3), 79-94. https:// aprendeenlinea.udea.edu.co/revistas/index.php/ ikala/article/view/14080 
Poehner, M. E., Davin, K. J. y Lantolf, J. P. (2017). Dynamic assessment. En E. Shohamy, I. G. Or y S. May (Eds.), Encyclopedia of Language and Education, Vol 7 (3a., pp. 243-256). Springer.

QSR International. (2002). NVivo. (Versión \# 12) [Software de computador]. https://www.qsrinternational.com/ nvivo-qualitative-data-analysis-software/supportservices/nvivo-downloads

Shohamy, E. (1998). Evaluation of learning outcomes in SLA Multiplism Perspective. In Learning foreign and second languages: Perspectives in research and scholarship, (pp. 238-2641). http://www.coh. arizona.edu/ classes/ariew/slat596/ Shohamy.pdf

Shohamy, E. (2001). Democratic assessment as an alternative. Language Testing, 18(4), 373-391. $\quad$ https://doi.org/https://doi. org/10.1177/026553220101800404

Stake, R. (1995). The art of case study research. Sage.
Suskie, L. (2002). Fair assessment practices: Giving students equitable opportunities to demonstrate learning. Adventures in Assessment, 14, 5-10. https://files.eric.ed.gov/fulltext/ED482885.pdf

Universidad de Antioquia. (2014). Acuerdo Académico 467 del 04 de diciembre de 2014. https://www.udea. edu.co/wps/wcm/connect/udea/3998a7c2-8f9b47d3-b9b6-6fe27fe96d45/+Acad\%C3\%A9mico+46 7+pol\%C3\%ADtica+de+competencia+en+lengua textranjera.pdf?MOD=AJPERES

Usma Wilches, J. A. (2009). Education and language policy in Colombia: exploring processes of inclusion, exclusion, and stratification in times of global reform. Profile Issues in Teachers' Professional Development, 11(1), 123-141. https://revistas.unal.edu.co/index. php/profile/article/view/10551/11014

Wiliam, D. (2011). What is assessment for learning? Studies in Educational Evaluation, 37(1), 3-14. https://doi.org/10.1016/j.stueduc.2011.03.001 